\title{
Perfil profesional de los educadores de deporte escolar en Bogotá Professional profile of school sport educators in Bogota
}

\author{
*Carlos Gutiérrez-García, **Luz Amelia Hoyos Cuartas, ***Monica Yuri Takito y*Ángel Pérez-Pueyo \\ * Universidad de León (España), ** Universidad Pedagógica Nacional (Bogotá, Colombia), *** Universidade de São Paulo (Brasil)
}

\begin{abstract}
Resumen: Este estudio tuvo como objetivo principal analizar el perfil profesional de los educadores deportivos que trabajan en programas de deporte escolar en Bogotá, y secundariamente contrastarlo con el perfil de los educadores deportivos españoles que muestran otros estudios. Se contó con una muestra de 233 educadores de instituciones educativas públicas y privadas y de los seis estratos socioeconómicos reconocidos en Colombia. Para la recogida de datos se utilizó el Cuestionario para la caracterización del deporte escolar en Bogotá (CCDE). Los datos fueron analizados mediante estadística descriptiva y test paramétricos y no paramétricos, dependiendo de su naturaleza. Los resultados mostraron el siguiente perfil general: hombre, edad media de 37 años, formación académica inicial a nivel de licenciatura, que realiza habitualmente actividades de formación continua, acumula entre 6 y 20 años de experiencia total en la profesión, y entre 1 y 10 en su institución actual. Los contratos más frecuentes son el anual y el indefinido, a tiempo completo. Los salarios presentaron importantes variaciones, y la mayor parte de educadores trabajaban también como profesores de educación física. Es precisamente este último hecho el que hace que existan importantes diferencias en el perfil profesional de los educadores deportivos bogotanos y españoles en la gran mayoría de aspectos analizados. Se concluye que no es posible definir claramente una carrera profesional para los educadores deportivos bogotanos, al igual que sucede en España.
\end{abstract}

Palabras clave: Deporte escolar, deporte educativo, educadores deportivos, profesores, entrenadores, monitores, técnicos deportivos, carrera profesional.

\begin{abstract}
This study aimed mainly to analyze the professional profile of sports educators working in school sports programs in Bogota, and secondarily to compare it with the profile of sport educators in Spain as it is shown in other studies. The sample was constituted by 233 educators of public and private educational institutions and the six socioeconomic strata recognized in Colombia. The Questionnaire for Characterization of School Sports in Bogota (QCSS) was used for data collection. Data were analyzed using descriptive statistics and a variety of parametric and nonparametric tests, depending on the nature of the data. The results showed the following general profile: male, mean age of 37 years, initial academic training at the graduate level, which typically does continuing education activities, has between 6 and 20 years of experience as school sports educator, and between 1 and 10 in his current position. The most common contracts are annual full-time and full-time indefinite. Salaries varied widely, and the majority of educators also worked as physical education teachers in the same institution. It is precisely this latter fact what differentiates, in most of the analysed factors, the profile of school sport educators in Bogota with respect to the profile of sport educators in Spain. We conclude that it is not possible to clearly define a career for sports educators of Bogota, as it also happen in Spain.
\end{abstract}

Key words: School sports, educational sport, sport educators, teachers, coaches, instructors, career.

\section{Introducción}

El deporte escolar, entendido como instrumento educativo, goza de un amplio consenso institucional a nivel internacional, que se ha plasmado en importantes documentos como la Carta Internacional de la Educación Física y del Deporte, promulgada por la UNESCO en 1978, la Carta Europea del Deporte y el Manifiesto sobre Jóvenes y Deporte, promulgados por el Consejo de Europa en 1992 y 1995 respectivamente, o la Carta Iberoamericana del Deporte, promulgada por el Consejo Iberoamericano del Deporte en 1996, entre otros (Orts, 2013). Particularmente en Colombia, la actual Constitución Política incluye al deporte como parte de la educación y lo considera un derecho social, económico y cultural orientado a la formación integral del ser humano y a la mejora y mantenimiento de la salud (Constitución Política de Colombia, art. 52). Desarrollando esta norma, la Ley 181, General del Deporte establece que el deporte es una conducta humana con características lúdicas y competitivas, que se realiza a través del ejercicio corporal y mental, y que está sujeto a normas que buscan generar valores morales, cívicos y sociales (art. 15). Asimismo, categoriza ocho formas de desarrollo del deporte: formativo, social comunitario, universitario, asociado, competitivo, de alto rendimiento, aficionado y profesional. Dentro de esta clasificación, el deporte escolar se incluye en el deporte formativo, el cual comprende los procesos de iniciación, fundamentación y perfeccionamiento deportivos (art. 16).

En la misma línea, existe un acuerdo mayoritario en el ámbito académico sobre la dimensión y potencial educativo del deporte escolar siempre que se creen las condiciones adecuadas (Blázquez, 1999;

\footnotetext{
Fecha recepción: 22-09-13- Fecha envío revisores: 28-09-13- Fecha de aceptación: 17-12-13 Carlos Gutiérrez-García

Campus de Vegazana s/n.

24071 - León. España.

carlos.gutierrez@unileon.es
}

Carreres, Escartí, Cortell, Fuster, \& Andreu, 2012; Fraile, 2004b; FraserThomas, Côté, \& Deakin, 2005; Gutiérrez, 2004; Hoyos, GutiérrezGarcía, Pérez-Pueyo, 2011; Petitpas, Cornelius, Van Raalte, \& Jones, 2005; Petrus, 1996; Velázquez, 2002). Por el contrario, también existe una importante evidencia de que una práctica deportiva mal orientada puede tener efectos perjudiciales para los jóvenes (Fraser-Thomas, et al., 2005; Hernández \& Velázquez, 1996; Petitpas, et al., 2005). Como señalan Carreres, et al. (2012), lejos de existir una relativa homogeneidad, la calidad de unos programas de deporte escolar (en adelante PDE) respecto a otros puede variar dramáticamente.

Dentro de los diversos actores que participan del deporte escolar (niños y jóvenes, educadores deportivos, coordinadores, árbitros, familias, instituciones escolares y deportivas, etc.), numerosos autores han destacado la importancia de aquellos que implementan en sí los programas (Álamo \& Amador, 2010; Cruz, Valiente, Mimbrero, Torregrosa, \& Boixadós, 1998; Fraile, 2004a; Fraser-Thomas, etal., 2005; Gutiérrez, 1995; Moreno \& Del Villar, 2004), y que aquí denominaremos educadores deportivos siguiendo a Maiztegui y Pereda (2000). Estas autoras defienden este término frente a otros habitualmente utilizados como monitor, entrenador, técnico, profesor o animador, ya que supone «el reconocimiento de una labor educativa realizada en contacto directo con los jóvenes deportistas» (p. 33). Tal y como recoge Delgado (2001, p.70), son funciones del educador deportivo:

- Dirigir las sesiones de entrenamiento.

- Enseñar conocimientos, conceptos e ideas.

- Crear un enfoque o clima positivo.

- Tomar decisiones y establecer normas.

- Liderar a los deportistas.

- Resolver temas administrativos o burocráticos de la competición.

- Establecer las relaciones públicas y sociales adecuadas en su entorno.

- Aconsejar a los deportistas. 
- Apoyar a los deportistas dentro y fuera del equipo.

- Analizar las actuaciones o comportamientos propios y relacionados con el equipo.

- Evaluar y tomar decisiones sobre los comportamientos de los deportistas.

- Relacionarse con el equipo directivo o responsables del equipo.

- Tener una actitud de continua actualización.

Por ello, autores como López (2000), Delgado (2001, 2004), o Carratalá (2011) han dibujado un amplio perfil de competencias y/o habilidades propias del educador deportivo. Como ejemplo, Delgado (2004) señala las siguientes competencias: (a) Como persona; (b) Para el logro; (c) Operativas de comunicación; (d) Interpersonales; (e) Directivas; (f) Técnicas; y (g) Otras. Tal exigencia ha llevado a proponer a los titulados superiores (en España, maestros especialistas en educación física y licenciados en ciencias de la actividad física y del deporteen adelante CCAFD -), como los profesionales más capacitados para actuar como educadores deportivos debido a su amplia formación pedagógica (Álamo, 2007; Fraile, 2000, 2004a). Por motivos similares, también se ha propuesto la formación del profesorado de educación física como base de la formación del educador deportivo (Moreno, 1997; Rodríguez, Castillo, \& Jiménez, 2001), o que los educadores deportivos hayan de complementar su educación formal con alguna titulación deportiva (Manrique, Gea, \& Álvaro, 2013).

A pesar de la importancia que se le reconoce a la figura del educador deportivo, y hasta donde llega nuestro conocimiento, no existe ningún estudio específico sobre su perfil formativo y profesional en Colombia (Hoyos, 2012). Ello contrasta con el caso español, donde se vienen realizando estudios desde mediados de la década de 1990 (e.g., Álamo, 2004; Álamo \& Amador, 2010; Gallardo-Pérez, Espada-Mateos, Santacruz-Lozano, \& Clemente-Remon, 2013; González, 1998; González, 2008; Jiménez, 2000; Manrique, et al., 2013; Nuviala, 2003; Nuviala, León, Gálvez, \& Fernández, 2007; Saura, 1996). De estas investigaciones se desprende la importancia del análisis del perfil del educador deportivo, tanto en términos diagnósticos - i.e., detectar la realidad un colectivo que habitualmente se ha considerado como escasamente formado (Álamo, 2007; Castejón, 2008; Fraile, 2000, 2004a; Fraser-Thomas, et al., 2005; González \& Campos, 2010; Nuviala, et al., 2007; Parker, 2000) -, como para informar políticas de actuación que promuevan su mejor formación y situación laboral. Igualmente, el perfil profesional de los educadores deportivos se ha definido como una demanda prioritaria del resto de agentes implicados en el deporte escolar (Asenjo \& Maiztegui, 2000; Gutiérrez, Carratalá, Guzmán, \& Pablos, 2010; Manrique, López-Pastor, Monjas, Barba, \& Gea, 2011).

Partiendo de estas consideraciones, el presente estudio tuvo como objetivos: (a) analizar el perfil demográfico, formativo y laboral de los educadores deportivos que trabajan en programas de deporte escolar (PDE) en Bogotá; (b) analizar las posibles diferencias existentes en estos perfiles según el carácter público o privado y según los estratos socio-económicos de las instituciones educativas escolares (en adelante IEE) donde estos educadores desarrollaban su labor; y (c) secundariamente, contrastar el perfil de estos educadores con el que muestran diversos estudios realizados en España (localizados a través de la bases de datos DIALNET y SPORTDiscus, el buscador Google Scholar y el catálogo de las bibliotecas universitarias españolas REBIUN), para establecer así un ámbito comparativo de referencia.

\section{Método}

\section{Población y muestra}

La población objeto de estudio fueron los educadores deportivos que trabajaban en PDE en Bogotá. Estos PDE debían cumplir los siguientes requisitos: (a) Ser ofertados por IEE públicas o privadas que atendían a población escolar de Bogotá, y que participaban en sus organizaciones y/o torneos escolares; (b) Ser desarrollados fuera de la clase de educación física; (c) Sin importar la finalidad de los mismos (lúdica, recreativa, competitiva, etc.), este factor no debía tenerse en cuenta; (d) Dirigidos por maestros, entrenadores, monitores, etc., o persona adulta responsable de la actividad; y (e) La participación debía ser voluntaria para los niños y jóvenes.

Sobre una población estimada de 1484 entrenadores (1313 de IEE privadas y 171 de IEE públicas) (Hoyos, 2012), y a partir de diversas acciones de difusión del proyecto y del contacto con las organizaciones responsables del deporte escolar en Bogotá, se conformó una muestra intencional basada en la voluntariedad de participación (Anguera, 2003), de 233 educadores deportivos ( NC 95\% $=5.9 \%$ ), en la que estuvieron representados entrenadores de IEE públicas $(n=51)$ y privadas $(n=$ 182). En Colombia, las IEE públicas son propiedad del Estado y dependen totalmente de sus recursos para su funcionamiento, mientras que las IEE privadas, mucho más numerosas, pertenecen a particulares y el Estado cumple únicamente una función de regulación y control. Del mismo modo, estuvieron representados educadores de IEE de todos los estratos socio-económicos. En relación a este término de estratos socioeconómicos, hemos de señalar que en Colombia es utilizado para categorizar a la población en función de sus ingresos económicos y condiciones de vida. Sediferencian seis estratos socio-económicos, siendo el 1 el que presenta más carencias y el 6 el más privilegiado. Las IEE atienden población de diversos estratos, pero principalmente a alumnos de varios estratos socio-económicos, altos, medios o bajos, y es un factor importante que define a las IEE. Para este estudio, dichos estratos fueron agrupados en tres niveles $A, B$ y C $\left(n_{A}=57 ; n_{B}=80 ; n_{C}=96\right)$, con mayor presencia de estratos bajos, medios y altos respectivamente.

Todos los educadores fueron informados previamente sobre los objetivos del estudio y el procedimiento para su participación en el mismo. Igualmente, se les garantizó la confidencialidad en el tratamiento de los datos obtenidos.

\section{Instrumentos}

Esta investigación formó parte de un proyecto más amplio orientado a analizar los PDE en Bogotá desde un punto de vista fundamentalmente descriptivo y cuantitativo. En dicho proyecto se diseñó y validó en tres fases (verificación de los propios autores, juicio de expertos y prueba piloto a educadores deportivos mediante el método test-retest) un cuestionario específico de 106 preguntas, principalmente de selección múltiple, denominado Cuestionario para la caracterización del deporte escolar en Bogotá (CCDE) (Hoyos, 2012; Hoyos, GutiérrezGarcía, \& Pérez-Pueyo, 2012), 24 de las cuales estaban dirigidas a los responsables instituciones del programa en la IEE (jefes del área de educación física o miembros del equipo directivo), y las 82 restantes debían ser respondidas por los educadores deportivos (CCDE). Entre las mismas, 16 hacen referencia a su perfil profesional, incluyendo aspectos relativos a formación académica inicial a nivel de pregrado y postgrado, formación permanente, experiencia y situación laboral. El resto de preguntas hacen referencia a aspectos generales del PDE, caracterización del PDE desde su diseño, y a las relaciones del PDE con la clase de educación física y con el proyecto educativo de la institución. El CCDE está diseñado para aplicarse mediante entrevista personal en un tiempo aproximado de 35 minutos.

\section{Procedimiento}

Las entrevistas fueron concertadas previamente con cada educador, y se realizaron entre mayo y diciembre de 2010, lo cual permitió la cobertura de las IEE bogotanas de calendario Ay B (curso académico de febrero a noviembre y de agosto a junio respectivamente).

\section{Análisis de datos}

Los datos obtenidos fueron analizados mediante el paquete estadístico STATA/IC 10. Se realizó en primer lugar una estadística descriptiva. Se utilizó el t test para analizar las diferencias entre educadores de IEE públicas y educadores de IEE privadas, en variables continuas que mostraron una distribución normal. Del mismo modo, se utilizó el test ANOVA para el análisis de los estratos agrupados (A, B y C), en las variables continuas distribuidas normalmente (e.g., en ambos casos, 
edad de los educadores deportivos). Para el análisis de las variables ordinales o binarias se aplicaron los test no paramétricos de MannWhitney (diferencias públicas-privadas) y Kruskal-Wallis (diferencias entre estratos agrupados), así como para las variables continuas que no mostraron una distribución normal. El nivel de significación se estableció en $p>$.05. Por último, se utilizó el índice de correlación de Pearson para estudiar la relación del salario de los educadores con sus años de experiencia y con los títulos conseguidos.

\section{Resultados}

Con el objeto de que la exposición de los resultados sea lo más sintética posible, únicamente se hará referencia a las diferencias entre educadores deportivos de IEE públicas y privadas y de los estratos agrupados en los casos en los que se hallaron diferencias significativas.

\section{Características generales}

La muestra presentó más hombres $(n=181 ; 78 \%$ ) que mujeres ( $n$ $=52 ; 22 \%$ ). La edad media de los educadores deportivos fue de $37 \pm 8$ años $(\min =19, \max =62)$, siendo las mujeres ( $35 \pm 9$ años) más jóvenes que los hombres (38 \pm 8 años) $\left(t_{(22)}=2.251 ; p=.025\right)$.

\section{Formación académica inicial}

Un 94.8\% de educadores eran titulados en al menos un campo académico, siendo el resto estudiantes (4.3\%) o profesionales no titulados (.9\%). Como muestra la Tabla 1, el 82.4\% de los educadores poseían un título de licenciatura. Se encontraron diferencias significativas respecto a la presencia de licenciados (graduados o estudiantes) en IEE públicas $(n=48 ; 94.1 \%)$ y privadas $(n=153 ; 84.1 \%)\left(Z_{M-W}=2.067 ; p\right.$ $=.0387$ ). Asimismo, el $94.4 \%$ de los educadores cursaba o había cursado estudios afines a las ciencias de la actividad física y del deporte (CCAFD).

\section{Formación académica de postgrado}

Un total de 80 educadores deportivos (34.3\%) tenían al menos un título de especialista. Se identificaron 13 programas de especialización relacionados con las CCAFD, que poseían o cursaban un total de 58 educadores (24.9\%). Se encontraron diferencias significativas respecto a la presencia de especialistas en PDE de IEE públicas ( $n=25$; 49\%) o privadas $\left(n=55\right.$; 30.2\%) $\left(Z_{M-W}=2.494 ; p=.0126\right)$. Únicamente un educador tenía formación en el ámbito de las CCAFD a nivel de maestría, y no se identificó ningún participante con formación doctoral.

\section{Cursos de formación permanente}

El 90\% de los educadores deportivos $(n=210)$ había realizado cursos de formación permanente, organizados por diversas personas o instituciones (entrenadores o expertos en un deporte, asociaciones de colegios privados, IDRD, federaciones deportivas, Coldeportes, universidades, las propias IEE, otras instituciones). Se hallaron diferencias significativas entre la realización de cursos entre educadores de IEE

\begin{tabular}{|c|c|c|c|c|c|c|c|c|c|c|}
\hline & \multicolumn{2}{|c|}{ Total } & \multicolumn{2}{|c|}{ Titulados } & \multicolumn{2}{|c|}{ Es tudiantes } & \multicolumn{2}{|c|}{ Afines* } & \multicolumn{2}{|c|}{ No afines* } \\
\hline & $n$ & $\%$ & $n$ & $\%$ & $n$ & $\%$ & $n$ & $\%$ & & $\%$ \\
\hline Licenciado & 201 & 86.3 & 192 & 82.4 & 9 & 3.9 & 193 & 82.8 & 8 & 3.4 \\
\hline Profesional & 39 & 16.7 & 32 & 13.7 & 7 & 3.0 & 25 & 10.7 & 14 & 6.0 \\
\hline Técnico & 14 & 6.0 & 13 & 5.6 & 1 & .4 & 9 & 3.9 & 5 & 2.1 \\
\hline Tecnólogo & 6 & 2.6 & 6 & 2.6 & 0 & .0 & 0 & 0 & 6 & 2.6 \\
\hline Otra titul ación & 6 & 2.6 & 4 & 1.7 & 2 & .9 & 4 & 1.7 & 2 & .9 \\
\hline Sin titulación & 2 & .9 & 0 & 0 & 0 & 0 & 0 & 0 & 0 & 0 \\
\hline
\end{tabular}
de una titulación. *Afinidad o no de los estudios cursados con el ámbito de las ciencias de la act ividad física y del deporte. Según la legislación colombiana sed efinen tres ciclos de formación para los program as de educación superior a nivel de pregrado. Estos son: (a) Técnico, primer ciclo, entre 1.5 y 2 años; (b) Tecnológico, segundo ciclo, entre 2.5 y 3.5 años; y (c) Profesi onal, tercer ciclo, normalmente de años. Las licenciaturas son formación de tercer ciclo orient adas a la capacitación docente.

Tabla 2. Años de experiencia y vinculación lab oral en la institución educativa escolar actual de los educadores deportivos

\begin{tabular}{lcccc} 
& \multicolumn{2}{c}{ Experiencia total } & \multicolumn{2}{c}{ En IEE actual } \\
& $n$ & $\%$ & $n$ & $\%$ \\
\hline 1 a 5 años & 42 & 18.0 & 136 & 58.3 \\
6 1 10 años & 62 & 26.6 & 49 & 21.0 \\
11 a 20 años & 90 & 38.6 & 37 & 15.8 \\
21 a 30 años & 32 & 13.7 & 9 & 3.8 \\
más de 30 años & 7 & 3.0 & 2 & .8 \\
TOTAL & 233 & 100 & 233 & 100 \\
\hline
\end{tabular}

públicas $(n=41 ; 80,4 \%)$ y privadas $(n=169 ; 92.9 \%)\left(Z_{M-W}=-2.632\right.$; $p=.0085)$ y de IEE de los estratos agrupados $\left(\mathrm{n}_{\mathrm{A}}=45 ; 78.9 \% ; \mathrm{n}_{\mathrm{B}}=73\right.$; $\left.91.3 \% ; \mathrm{n}_{\mathrm{C}}=92 ; 95.8 \%\right)\left(x_{K-W}^{2}=11.584 ; p=.0032\right)$. La media de cursos totales realizados fue de $10 \pm 10(\mathrm{M} \pm \mathrm{DE})$, y las frecuencias más habituales fueron una o dos veces al año (55\% y $21 \%$ respectivamente).

\section{Actividades complementarias de formación}

Los educadores deportivos asignaron una valoración, a través de una escala tipo Likert de 5 niveles (ninguna a mucha importancia), a las actividades complementarias que realizaban autónomamente para su formación permanente. La actividad considerada más importante fue la consulta de páginas de internet $(M=4.2 ; D E=1.0)$, seguida de la compra o consulta de bibliografía $(M=3.8 ; D E=.9)$ y la asistencia a seminarios y congresos $(M=3.6 ; D E=1.1)$. La valoración de las consultas de páginas en internet fue significativamente mayor que la atribuida a la consulta de bibliografía $\left(Z_{M-W}=5.455 ; p<.001\right)$ y a la asistencia seminarios $\left(Z_{M-W}=7.085 ; p<.001\right)$, así como la valoración de la consulta de bibliografía fue mayor que la de asistencia a seminarios $(Z$ $\left.{ }_{M-W}=2.671 ; p=.0076\right)$. Un total de 76 educadores (32.6\%) mencionaron otras actividades complementarias de formación, considerándolas en conjunto entre bastante y muy importantes $(M=4.3 ; D E=.6)$. Entre estas actividades figuraban, de mayor a menor frecuencia, dialogar con colegas y entrenadores para compartir conocimientos, participar en torneos, practicar el deporte, observar entrenamientos o competiciones, y realizar trabajos escritos o audiovisuales.

\section{Experiencia y vinculación laboral}

La experiencia de los educadores deportivos en el desarrollo de PDE se situó entre 1 y 45 años $(M=13 ; D E=8)$, mientras que en relación a los años de vinculación con la IEE actual los márgenes se situaron entre 1 y 38 años $(M=7 ; D E=7)$ (Tabla 2).

Con respecto al tipo de vinculación, se encontró una amplia variedad de tipos de contratos (Tabla 3). Los más frecuentes fueron el contrato anual a tiempo completo - esto es, el contrato tiene que renovarse cada año para que el educador pueda continuar su labor-(59.7\%) y el contrato indefinido a tiempo completo-más estable que el anterior, en el que existe una vinculación permanente entre el educador y la IEE - (15.9\%). La categoría «otros» incluyó fundamentalmente contratos de orden de prestación de servicios. Dos educadores trabajaban en IEE de forma voluntaria, y otro trabajaba sin contrato en una IEE privada. Se hallaron diferencias significativas en todos los tipos de contrato respecto al carácter y estratos agrupados de las IEE (Tabla III).

\section{Salario}

El 65.7\% de los educadores deportivos $(n=153)$ informó sobre su salario mensual en el PDE. El 88.4\% de los educadores tenía otro u otros empleos además del PDE, mientras que para el 9.0\% era su única fuente de ingresos (un 2.6\% no respondió a la pregunta). Dehecho, el 73.8\% de los educadores manifestaron tener otro empleo como profesores de la educación física escolar en la misma IEE donde desarrollaban el PDE.

\begin{tabular}{|c|c|c|c|c|c|c|c|c|c|c|c|}
\hline & $\%$ total & Carácter & $n$ & $\%$ & $\frac{\text { Mann- }}{Z}$ & Nhi tney & EA & $n$ & $\%$ & $\begin{array}{l}\text { Kruskal } \\
?^{2}\end{array}$ & $\begin{array}{l}\text { al-Wallis } \\
\text { gl }\end{array}$ \\
\hline Completo & 59.7 & $\begin{array}{l}\text { Públicas } \\
\text { Privadas }\end{array}$ & $\begin{array}{c}7 \\
132\end{array}$ & $\begin{array}{l}13,7 \\
72.5\end{array}$ & -7.549 & .000 & $\begin{array}{l}\text { A } \\
\text { B } \\
\text { C }\end{array}$ & $\begin{array}{l}12 \\
63 \\
64\end{array}$ & $\begin{array}{l}21.1 \\
78.8 \\
66.7\end{array}$ & 49.161 & \\
\hline Completo indefinido & 15.9 & $\begin{array}{l}\text { Públicas } \\
\text { Privadas }\end{array}$ & $\begin{array}{l}24 \\
13\end{array}$ & $\begin{array}{l}47.1 \\
7.1\end{array}$ & -6.878 & .000 & $\begin{array}{l}\text { A } \\
\text { B } \\
\text { C }\end{array}$ & $\begin{array}{c}21 \\
5 \\
11\end{array}$ & $\begin{array}{c}36.8 \\
6.3 \\
11.5\end{array}$ & 25.598 & 2.000 \\
\hline Otros & 11.6 & $\begin{array}{l}\text { Públicas } \\
\text { Privadas }\end{array}$ & $\begin{array}{l}17 \\
10\end{array}$ & $\begin{array}{l}33.3 \\
5.5 \\
\end{array}$ & -5.478 & .000 & $\begin{array}{l}\text { A } \\
\text { B } \\
\text { C }\end{array}$ & $\begin{array}{c}20 \\
3 \\
4 \\
\end{array}$ & $\begin{array}{c}35.1 \\
3.8 \\
4.2 \\
\end{array}$ & \multicolumn{2}{|c|}{$40.508 \quad 2$} \\
\hline Por horas & 11.6 & $\begin{array}{l}\text { Públicas } \\
\text { Privadas }\end{array}$ & $\begin{array}{c}1 \\
26\end{array}$ & $\begin{array}{l}2.0 \\
14.3\end{array}$ & -2.425 & .015 & $\begin{array}{l}\text { A } \\
\text { B } \\
\text { C }\end{array}$ & $\begin{array}{c}2 \\
8 \\
17\end{array}$ & $\begin{array}{c}3.5 \\
10.0 \\
1.7\end{array}$ & \multicolumn{2}{|l|}{7.307} \\
\hline \multicolumn{12}{|c|}{$\begin{array}{l}\text { \% total = porcentaje total de educadores deportivos segú } n \text { tipo de contrato; } n=\text { nú mero de educadores; } \%= \\
\text { porcentaje de educadores en su cat egoría. EA = Estratos agupados. } n \text { to tal }=233 ; n_{\text {IEEpúb }}=51 ; n_{\mathbb{E E F} \text { Friv }}=182 ; \\
\mathrm{n}_{\mathrm{A}}=57 ; \mathrm{n}_{\mathrm{B}}=80 ; \mathrm{n}_{\mathrm{C}}=96 \text {. }\end{array}$} \\
\hline \multirow{2}{*}{\multicolumn{3}{|c|}{$\begin{array}{l}\text { Tabla 4. Medianas y percentiles } \\
\text { Educadores deportivos de IEE }\end{array}$}} & \multirow{2}{*}{\multicolumn{3}{|c|}{$\frac{\text { los edu }}{M d n}$}} & \multirow{2}{*}{\multicolumn{3}{|c|}{$\frac{\text { portivos }}{\text { Percentil } 25}$}} & \multirow{2}{*}{\multicolumn{3}{|c|}{$\begin{array}{l}\text { Percent il } 75 \\
\$ 900000(350 €)\end{array}$}} \\
\hline & & & $\$ 900000(350 €)$ & & & & & $\begin{array}{l}\text { Percentll 2S } \\
\$ 300000(117 €)\end{array}$ & & & \\
\hline & & & \multirow{2}{*}{\multicolumn{3}{|c|}{$\begin{array}{l}\$ 600000(234 €) \\
\$ 850000(331 €)\end{array}$}} & \multirow{2}{*}{\multicolumn{3}{|c|}{$\$ 405000(158 €)$}} & \multicolumn{3}{|c|}{$\begin{array}{c}\$ 900000(350 €) \\
\$ 1000000(390 €)\end{array}$} \\
\hline \multicolumn{3}{|l|}{ pivadato agrupado A } & & & & & & & \multicolumn{3}{|c|}{$\frac{\$ 10000000(390 €)}{\$ 90000(350 €)}$} \\
\hline \multirow{2}{*}{\multicolumn{3}{|c|}{ estrato agrupado B }} & \multicolumn{3}{|c|}{$\$ 600000$ (234€) } & & $0(156$ & & & & \\
\hline & & & & & & & & & & & \\
\hline Total & & & $\$ 600$ & $000(2$ & $34 €)$ & $\$ 400000$ & $0(156$ & & & $00000(35 \mathrm{C}$ & $50 €$ \\
\hline
\end{tabular}


El salario mensual de los educadores, recibido únicamente por su trabajo en el PDE, osciló entre \$60.000 y \$3.000.000 de pesos (23 y 1170 euros respectivamente; $\$ 1.000$ pesos colombianos equivalen a fecha de 15/09/2013 a .39 euros o .52 dólares estadounidenses), con una mediana de $\$ 600.000$ pesos (234 euros). La Tabla 4 presenta las medianas y percentiles de los salarios de los educadores según el carácter y el estrato agrupado de la IEE. La prueba de la mediana solo mostró diferencias significativas según el carácter de la IEE, a favor de los educadores deportivos de las IEE públicas $\left(x_{(1)}^{2}=5.327 ; p=.036\right)$.

Al relacionar la experiencia de los educadores con su salario mensual, se encontró una baja correlación con los años totales de experiencia $\left(r=.184 ; r^{2}=.033\right)$, y con los años de experiencia en su IEE actual ( $r=$ $\left..13 ; r^{2}=.01\right)$.

\section{Títulos obtenidos en torneos de deporte escolar}

Un total de 166 entrenadores (71.2\%) señalaron haber obtenido al menos un título en torneos de deporte escolar en los tres últimos años con alguno de sus equipos (primer, segundo o tercer puesto). La media de títulos conseguidos fue de $7 \pm 6(\mathrm{M} \pm \mathrm{DE})(\min =1$; máx $=36)$. Se observaron diferencias significativas en el número de títulos conseguidos en función de los estratos agrupados de las IEE $\left(\mathrm{M} \pm \mathrm{DE}_{\mathrm{A}}=6.8 \pm\right.$ 4.8; $\left.\mathrm{M} \pm \mathrm{DE}_{\mathrm{B}}=8.7 \pm 6.5 ; \mathrm{M} \pm \mathrm{DE}_{\mathrm{C}}=6.3 \pm 7.0\right)\left(x^{2}{ }_{K-W}=11.038 ; p=\right.$ .004). La correlación encontrada entre el número de títulos conseguido por los entrenadores y su salario fue muy baja $\left(r=.094 ; r^{2}=.009\right)$.

\section{Discusión}

Los resultados obtenidos muestran que el campo profesional del deporte escolar en Bogotá es desarrollado principalmente por hombres, en una relación de 3:1 respecto a las mujeres. Este desequilibrio concuerda con las cifras históricas de formación de profesionales de educación física y áreas afines en Bogotá, que muestran una proporción de 70 $30 \%$ favorable a los hombres (cifra estimada a partir de los graduados de la Facultad de Educación Física de la Universidad Pedagógica Nacional en Bogotá), y es similar al descrito en diversos estudios realizados en España (Espada \& Calero, 2010; Gallardo-Pérez, et al., 2013; Jiménez, 2000; Manrique, et al., 2013; Nuviala, et al., 2007), validando en este ámbito las tesis de Hargreaves (1986) o Wenner y Jackson (2009), quienes consideran al deporte como un campo originalmente masculino y fundamentalmente asociado a la definición cultural de la masculinidad. A pesar de ello, la menor edad media de las mujeres podría indicar una posible mayor presencia de mujeres educadoras de deporte escolar en el futuro.

La edad media de los educadores fue de 37 años, y es elevada en comparación con los datos aportados por diversos estudios realizados en España, que sitúan a la mayor parte de educadores deportivos por debajo de los 25 o 30 años (Álamo, 2004; Álamo \& Amador, 2010; Espada \& Calero, 2010; Gallardo-Pérez, et al., 2013; Manrique, et al., 2013; Nuviala, et al., 2007). Un importante factor explicativo de la alta edad media de los entrenadores bogotanos es que en su mayoría estos trabajan también como profesores de educación física de las IEE donde desarrollan sus PDE, mientras que en el caso español, como señalaremos más adelante, esta circunstancia ya no es frecuente.

Por lo que se refiere a la formación académica, el 94\% de los educadores deportivos manifestó realizar o haber realizado estudios relacionados con las ciencias de la actividad física y del deporte, el 83\% poseía una formación específica a nivel de licenciatura y el 25\%, además, a nivel de posgrado. Esta realidad contrasta con la que se vivió en Colombia en las décadas de 1950 y 1960, donde los encargados de desarrollar el deporte en la escuela fueron deportistas - en activo o retirados-, padres de familia o voluntarios (Gómez \& Parra, 1986). A partir de los años 70, el deporte comenzó a tener un lugar más destacado en la escuela en perjuicio de la gimnasia (Pinillos, 2006), y los licenciados comenzaron a ocupar el espacio que antes ocuparan personas con menor cualificación. En la actualidad, tal y como reflejan nuestros resultados, las IEE mantienen un alto nivel de exigencia respecto a la formación inicial de los educadores deportivos, lo cual podría favorecer una mejor calidad de los programas y una mayor adhesión a la práctica por parte de los jóvenes (González \& Campos, 2010; Nuviala, 2003; Rodríguez, et al., 2001). La situación española es bastante diferente; la formación que poseen los educadores deportivos suele ser más elemental (Álamo, 2004; Álamo, Amador, \& Pintor, 2002a; Delgado, 2001; Manrique, etal., 2013; Nuviala, et al., 2007), está ligada generalmente a las titulaciones que expiden las federaciones deportivas (Álamo \& Amador, 2010; Corral, 2007; Espada \& Calero, 2010; Jiménez, 2000; Nuviala, et al., 2007; Orts, 2013), o es inexistente hasta en un 50\% de los casos (Martínez, Campos, Pablos, \& Mestre, 2008). Con todo, en nuestro estudio sigue existiendo un cierto grado de intrusismo profesional ligeramente superior al 5\%, debido a la carencia de una regulación específica al respecto, lo cual podría condicionar la calidad de algunos PDE (Fraile, Arribas, Gutiérrez, \& Hernández, 1998; Kirk \& Monjas, 2004; Manrique, et al., 2011).

En cualquier caso, el factor explicativo fundamental de la alta cualificación de los educadores deportivos bogotanos es, nuevamente, que el 71\% de los mismos son también profesores de la materia de educación física en su IEE. Esta situación difiere con lo que sucede en España, donde el profesorado de educación física escolar raramente se implica en actividades extraescolares (Álamo, 2004; Delgado, 2001; González \& Campos, 2010; Velázquez, Devís, de la Torre, Carranza, \& Fraile, 2004).

Por otra parte, las diferencias entre educadores deportivos de IEE públicas y privadas respecto a la posesión de títulos de posgrado puede explicarse a partir de la normativa reguladora de la función docente en las IEE públicas, donde la formación postgradual es uno de los criterios tenidos en cuenta para el ascenso en el escalafón salarial (Ministerio de Educación Nacional, 2002), lo cual hace que los profesionales vinculados al sector público se interesen por cursar programas de postgrado.

El alto nivel de la formación inicial de los educadores deportivos bogotanos se mantiene en el ámbito de la formación permanente, lo cual muestra una preocupación por estar actualizados y así mejorar su desempeño y cualificación profesional (Fernández, 2009). La diversidad de agentes implicados en la promoción de cursos de formación refleja el interés que por diversos motivos (pedagógicos, económicos, sociales, políticos, etc.) tienen estos agentes en la formación permanente de los educadores deportivos. En el caso español existe una importante disparidad de cifras, que en cualquier caso son inferiores a las registradas en nuestro estudio (Álamo, et al., 2002a; Corral, 2007).

También como parte de su formación permanente, los educadores deportivos destacaron la consulta de páginas de internet y el dialogo con otros entrenadores como las actividades de mayor importancia para actualizar conocimientos. Estos resultados son similares a los hallados por Nelson, Cushion y Potrac (2006) sobre las actividades informales que realizaban los entrenadores para adquirir conocimientos, aunque los resultados obtenidos en nuestro estudio pueden estar condicionados por no haber incluido ítems específicos sobre algunos de estos aspectos. En relación a la compra y/o consulta de bibliografía, y la asistencia a seminarios y congresos, estas actividades pueden tener asociadas algunas desventajas como un mayor coste económico, menor inmediatez y menor libertad para su realización, lo cual puede explicar sus menores puntuaciones.

En relación a la experiencia de los educadores deportivos, los 13 años encontrados de media son muy superior a descritos en diversos estudios realizados en España, en los que la experiencia media de los entrenadores suele ser inferior a tres años (Campos, 2005; GallardoPérez, et al., 2013; Manrique, et al., 2013; Nuviala, et al., 2007), y frecuentemente es solo es de un año. Así, como señala Jiménez (2000), los educadores deportivos en España son, en general, personas jóvenes que tienen este trabajo como temporal. En nuestro estudio, sin embargo, los educadores deportivos bogotanos mostraron una mayor experiencia debido a que en Colombia este trabajo está claramente asociado a la educación física escolar, circunstancia no generalizada en España. Los datos recogidos sobre los años de vinculación con su IEE actual muestran que, en general, los educadores deportivos bogotanos han trabajado, al menos, en dos IEE.

Los tipos de contrato de los educadores deportivos son muy variados; sin embargo, solamente un 16\% de los contratos son indefinidos, y 
estos se ofrecen mayoritariamente en las IEE públicas. El resto de contratos brindan poca seguridad laboral, ya que tanto los contratos anuales como los contratos por horas solo cubren los meses del año correspondientes al calendario escolar, que en general no supera los diez meses. No obstante, la situación que se dibuja en nuestro estudio es mucho mejor que la española, donde se han encontrado porcentajes de educadores deportivos trabajando sin contrato laboral superiores al 50\% (Campos, 2005; Nuviala, 2003), y hasta de más del 85\% (Gallardo-Pérez, et al., 2013), lo cual es una claro ejemplo de economía sumergida.

Así, en general, para la mayoría de los educadores deportivos su trabajo en el PDE era un trabajo auxiliar, que complementaba su trabajo como docente de educación física de la misma IEE, o que se combinaba con otra actividad laboral en otra institución. Como referencia, el salario medio de los educadores deportivos fue un $31 \%$ mayor que el salario mínimo legal vigente para Colombia, que fue de $\$ 576500$ pesos en el año 2010 (equivalentes aproximadamente a 215 euros). No obstante hemos de ser cautelosos con cualquier tipo de comparación ya que, como se ha señalado, se hallaron diferentes tipos de contrato, muchos de los cuales no eran a jornada completa. Otro valor de referencia puede ser el salario en una IEE pública para un licenciado, para el primer nivel de escalafón (el cual no requiere años de experiencia), que es de \$1262811 pesos (492 euros) (el escalafón docente para los maestros de IEE públicas puede consultarse en http://nominas.com.co/escala-salarialdocentes.html). Por ello, es muy habitual, como se ha señalado, que los educadores deportivos tengan otras ocupaciones laborales que complementen sus ingresos. Con todo, son reseñables las grandes diferencias salariales existentes. Por citar los ejemplos más extremos, un educador deportivo de una IEE privada de estrato 1 tenía un salario de \$60000 pesos mensuales (23 euros), mientras que otro que trabajaba en una IEE privada de estrato 6 ganaba $\$ 3000000$ pesos mensuales (1170 euros).

En un ámbito comparativo, el escaso salario recibido y las pocas perspectivas de desarrollar una carrera profesional en el deporte escolar han sido citados como importantes motivos para la no permanencia de los educadores deportivos en el deporte escolar en España (Campos, González, Pablos, \& Marín, 2007; Gallardo-Pérez, et al., 2013; Manrique, et al., 2013) y para la consideración de esta actividad laboral como secundaria a otra principal (Álamo, 2004; Álamo, Amador, \& Pintor, 2002b), por lo que su profesionalización aparece como un factor clave para la mejora de los programas de deporte escolar (Manrique, et al., 2011).

Por lo que se refiere a las diferencias entre los educadores deportivos de IEE públicas y privadas, los educadores de IEE públicas como colectivo presentaban un perfil formativo inicial más alto, tenían contratos más estables y mejor salario que los de las IEE privadas, que a su vez realizaban más cursos de formación permanente que los primeros. Se encontraron menos diferencias respecto a los estratos agrupados: los educadores de IEE de estratos agrupado B realizaban más cursos de formación permanente, y junto con los educadores del estrato agrupado A solían tener contratos completos anuales, mientras que los educadores de IEE de estrato agrupado Asolían tener contratos indefinidos y de prestación de servicios. Para explicar estas diferencias ha de considerarse que muchos educadores de IEE públicas están vinculados al sector público como profesores de educación física, y que este desempeño exige el cumplimiento de algunos requisitos como estar en posesión de un título de licenciado (Ministerio de Educación Nacional, 2002). Asimismo, gran parte de los contratos de los educadores de IEE públicas independientemente del estrato -, se rigen por el estatuto docente, reglamentado por el Ministerio de Educación Nacional, esto es, no negociados con la IEE como sucede más frecuentemente en las IEE privadas, y de ahí la mayor disparidad salarial que presentan estas últimas.

Finalmente, es importante señalar que ni los años de experiencia (totales y en la misma IEE) ni los éxitos deportivos logrados (medidos en términos de títulos deportivos) guardaron relación con el salario de los educadores deportivos, por ello parece que no es posible la definición clara de una carrera profesional en el oficio de educador deportivo, dado además queni en el sector público ni en el sector privado existe una reglamentación al respecto. A efectos prácticos, esto se traduce en que, generalmente, los educadores deportivos que quieren mejorar sus condiciones laborales y/o salario buscan IEE privadas de estratos socioeconómicos más altos que puedan satisfacer sus pretensiones.

\section{Conclusiones y limitaciones del estudio}

El 75\% de educadores deportivos que desarrollaban PDE en Bogotá son hombres, presentando el colectivo una edad media de 37 años. Son varios los indicadores que muestran que el perfil profesional de estos educadores deportivos es de un alto nivel: en general, estos educadores deportivos tienen una formación académica universitaria y específica, y realizan habitualmente actividades formales e informales para su formación permanente. Asimismo, acumulan una media de 7 años de experiencia en deporte escolar, habitualmente en más de una IEE. Estos datos contrastan con la realidad española, donde el perfil profesional del educador deportivo responde a una persona más joven, con un menor nivel formativo y menor experiencia.

No obstante, el buen nivel del perfil profesional de los educadores deportivos de Bogotá, presente tanto en IEE públicas como privadas y de diferentes estratos agrupados, no se ve acompañado en un ámbito contractual, salarial ni de carrera profesional. El trabajo como educador deportivo en PDE es mayoritariamente una dedicación secundaria a otra principal, habitualmente profesor de educación física de la misma IEE. No es posible definir claramente una carrera profesional para estos educadores deportivos, circunstancia similar a lo que sucede en España.

Respecto a las limitaciones del estudio, además de las ya señaladas ha de destacarse que las respuestas de los educadores deportivos podrían haberse visto condicionadas por su deseo de presentar una imagen favorable (Morales, 2006; Pozo et al., 2006) de ellos mismos, de su colectivo y/o de su IEE. En este sentido, la aplicación del cuestionario mediante entrevista individual trató de paliar este efecto y lograr una mayor calidad y espontaneidad en las respuestas (Cea D'ancona, 2001). En segundo lugar, este estudio se realizó sobre PDE ofertados por IEE que participaban en organizaciones y/o torneos de deporte escolar. Existen en Bogotá, y en general en Colombia, PDE que no participan de estas organizaciones o competiciones-i.e., PDE exclusivamente internos de cada IEE-, que no han podido ser conocidos ni tenidos en cuenta (Hoyos, 2012). La realización de un trabajo exploratorio de detección de estos PDE habría requerido un amplísimo estudio previo que no podía ser asumido por el equipo investigador.

\section{Referencias}

Álamo, J. M. (2004). El deporte escolar en Canarias. En A. Fraile (Ed.), El deporte escolar en el siglo XXI: Análisis y debate desde una perspectiva europea (pp. 133-152). Barcelona: Graó.

Álamo, J. M. (2007). Concepto, evolución y diferentes manifestaciones del deporte. La iniciación deportiva y el deporte escolar. Las Palmas de Gran Canaria: Anroart.

Álamo, J. M., \& Amador, F. (2010). El entrenador y la organización del deporte escolar. Tándem: Didáctica de la educación física, 32, 93-104.

Álamo, J. M., Amador, F., \& Pintor, P. (2002a). Funcion social del deporte escolar. El entrenador del deporte escolar. Lecturas: Educacion Fisica y Deportes, 45. Recuperado de: http://www.efdeportes.com/efd45/escolar.htm

Álamo, J. M., Amador, F., \& Pintor, P. (2002b). El deporte escolar: conquista de nuevos espacios en el mercado laboral. Revista Española de Educación Física y Deportes, 4, 5-10.

Anguera, M. T. (2003). La metodología selectiva en la Psicología del Deporte. En A. Hernández Mendo (Ed.), Psicología del Deporte (Vol. 2: Metodología, pp. 74-96). Buenos Aires: Efdeportes.

Asenjo, F., \& Maiztegui, C. (2000). La interrelación entre los distintos agentes implicados en el deporte escolar: un análisis de sus demandas desde el punto de vista de los educadores deportivos. En C. Maiztegui \& V. Pereda (Eds.), Ocio y deporte escolar (pp. 41-63). Bilbao: Universidad de Deusto.

Blázquez, D. (Ed.). (1999). La iniciación deportiva y el deporte escolar. Barcelona: Inde.

Campos, A. (2005). Situación profesional de las personas que trabajan en funciones de actividad física y deporte en la Comunidad Autónoma Valenciana (2004). Tesis Doctoral. Universitat de València, Valencia. Recuperado de: http:/hdl.handle.net/10803/9710 
Campos, A., González, M. D., Pablos, C., \& Marín, M. (2007). Los recursos humanos en el deporte escolar de los centros educativos de la Comunidad Valenciana. Revista de educación física: Renovar la teoría y practica, 105, 11 19.

Carratalá, V. (2011). Cualificación y perfil profesional en el deporte escolar Ponencia presentada en el II Congreso del deporte en Edad Escolar, Valencia, 26-28 de octubre. Recuperado de: http://www.deporteescolarvalencia.com/ uploads/editor/cualificacion_y_perfil_profesional.pdf

Carreres, F., Escartí, A., Cortell, J. M., Fuster, V., \& Andreu, E. (2012). The relationship between out-of-school sport participation and positive youth development. Journal of Human Sport and Exercise, 7(3), 671-683.

Castejón, F. J. (2008). Deporte escolar y competición. En A. I. Hernández, L. F. Martínez \& C. Águila (Eds.), El deporte escolar en la sociedad contemporánea (pp. 159-177). Almería: Universidad de Almería.

Cea D’ancona, M. A. (2001). Metodología cuantitativa. Estrategias y técnicas de investigación social. Madrid: Síntesis.

Corral, J.A. (2007). ¿Quién atiende el entrenamiento en el deporte en edad escolar? El perfil de los técnicos de las escuelas deportivas municipales. Paper presented at the II Congreso Internacional y XXIV Nacional de Educación Física, Palma de Mallorca. Recuperado de: www.uibcongres.org/imgdb//archivo_dpo2415.doc

Cruz, J., Valiente, L., Mimbrero, J., Torregrosa, M., \& Boixadós, M. (1998). Pape de los agentes de socialización en deportistas de edad escolar. Revista de psicología del deporte, 7(2), 295-310.

Delgado, M. A. (2001). El papel del entrenador en el deporte durante la edad escolar. Deporte y actividad física para todos, 2, 63-78.

Delgado, M. A. (2004). Prólogo. En M. P. Moreno \& F. Del Villar (Eds.), El entrenador deportivo: manual práctico para su desarrollo y formación (pp. 711). Barcelona: Inde.

Espada, M., \& Calero, J. C. (2010). Análisis de la evaluación del alumnado de educación secundaria en las actividades físico-deportivas extraescolares. ENSAYOS, Revista de la Facultad de Educación de Albacete, 25, 97-109.

Fernández, E. (2009). Formación y supervisión de los monitores de baloncesto, en las escuelas deportivas municipales de Aljaraque. Revista Wanceulen E.F. Digital, 5, 146-160. Recuperado de: http://hdl.handle.net/10272/3318

Fraile, A. (2000). Deporte escolar e instituciones públicas. En J. A. Párraga \& M. L. Zagalaz (Eds.), Reflexiones sobre educación física y deporte en la edad escolar (pp. 139-161). Jaén: Universidad de Jaén.

Fraile, A. (2004a). Hacia un deporte escolar educativo. EnA. Fraile(Ed.), El deporte escolar en el siglo XXI : análisis y debate desde una perspectiva europea (pp. 9-28). Barcelona: Graó.

Fraile, A. (Ed.). (2004b). El deporte escolar en el siglo XXI: análisis y debate desde una perspectiva europea. Barcelona: Graó.

Fraile, A., Arribas, H., Gutiérrez, S., \& Hernández, A. (1998). La salud en las actividades físicas extraescolares. En M. Santos \& A. Sicilia (Eds.), Activida des físicas extraescolares: Una propuesta alternativa (pp. 53-62). Barcelona: Inde.

Fraser-Thomas, J. L., Côté, J., \& Deakin, J. (2005). Youth sport programs: an avenue to foster positive youth development. Physical Education and Sport Pedagogy, 10(1), 19-40.

Gallardo-Pérez, J. M., Espada-Mateos, M., Santacruz-Lozano, J. A., \& ClementeRemon, A. L. (2013). Las características laborales del profesorado de las actividades físico-deportivas extraescolares en función de la edad y el sexo, dentro de la Comunidad de Madrid. RICYDE: Revista Internacional de Ciencias del Deporte, 9(31), 23-37.

Gómez, A., \& Parra, L. (1986). Historia de la educación física en Colombia como profesión. Bogotá: Editorial Universidad Central.

González, J.(1998). Organización deportiva extraescolar en los centros docentes de Barcelona y su cinturón. Diseño de un modelo. Tesis Doctoral. Universidad de Barcelona, Barcelona.

González, M. D. (2008). El deporte escolar en la comunidad autónoma de Madrid: Intervención didáctica y recursos humanos en las actividades físico-deportivas extraescolares en los centros educativos. Tesis Doctoral. Universidad de Valencia, Valencia.

González, M. D., \& Campos, A. (2010). La intervención didáctia del docente de deporte escolar, según su formación inicial. Revista de psicodidáctica, 15(1), 101-120.

Gutiérrez, M. (1995). Valores sociales y deporte. Madrid: Gymnos.

Gutiérrez, M. (2004). El valor del deporte en la educación integral del ser humano. Revista de educación, 335, 105-126.

Gutiérrez, M., Carratalá, V., Guzmán, J. F., \& Pablos, C. (2010). Objetivos y manifestación de valores sociales y personales en el deporte juvenil según deportistas, padres, entrenadores y gestores. Apunts: Educación física y deportes, 101, 57-65.

Hargreaves, J. (1986). Sport, power and culture. Cambrigde: Polity Press.

Hernández, J. L., \& Velázquez, R. (1996). La actividad física extraescolar en los centros educativos. Madrid: MEC.

Hoyos, L. A. (2012). Caracterización de los programas de deporte escolar en Bogotá. Análisis de los modelos didácticos empleados para su enseñanza. Tesis Doctoral. Universidad de León, León. Recuperado de: http://hdl.handle.net/ $10612 / 2315$
Hoyos, L. A., Gutiérrez-García, C., \& Pérez-Pueyo, A. (2011). Deporte escolar. Fundamentación teórica. Armenia (Colombia): Kinesis.

Hoyos, L. A., Gutiérrez-García, C., \& Pérez-Pueyo, A. (2012). Caracterización de los programas de deporte escolar en Bogotá. Revista Educación Física y Deporte, 31(1), 853-860.

Jiménez, D. (2000). El perfil profesional y didáctico de los técnicos de los deportes colectivos en la oferta pública de práctica deportiva en edad escolar. En VV.AA (Ed.), Actas del I Congreso Nacional de Deporte en Edad Escolar (pp. 435452). Sevilla: Ayuntamiento de Dos Hermanas.

Kirk, D., \& Monjas, R. (2004). El deporte escolar en el Reino Unido. En A. Fraile (Ed.), El deporte escolar en el siglo XXI : análisis y debate desde una perspectiva europea (pp. 49-58). Barcelona: Graó.

López, J. F. (2000). Las habilidades del educador deportivo. En C. Maiztegui Oñate \& V. Pereda Herrero (Eds.), Ocio y deporte escolar (pp. 91-111). Bilbao: Universidad de Deusto.

Maiztegui, C., \& Pereda, V. (Eds.). (2000). Ocio y deporte escolar. Bilbao: Universidad de Deusto.

Manrique, J. C., Gea, J. M. \& Álvaro, M. (2013). Perfil y expectativas del técnico de deporte escolar en el municipio de Segovia (España). RICYDE: Revista Internacional de Ciencias del Deporte, 50. Recuperado de: http:// dialnet.unirioja.es/servlet/extart?codigo $=4274634$

Manrique, J. C., López-Pastor, V. M., Monjas, R., Barba, J. J., \& Gea, J. M. (2011). Implantación de un proyecto de transformación social en Segovia (España): desarrollo de un programa de deporte escolar en toda la ciudad. Apunts: Educación física y deportes, 105, 58-66.

Martínez, G., Campos, A., Pablos, C., \& Mestre, J. A. (2008). Los recursos humanos de la actividad física y del deporte. Funciones y características socio-demográficas laborales y formativas. Valencia: Tirant lo Blanch.

Ministerio de Educación Nacional (2002). Decreto 1278 de Junio 19 de 2002, por el cual se expide el estatuto de profesionalización docente. Recuperado de: http:/ /www.mineducacion.gov.co/1621/articles-86102_archivo_pdf.pdf

Morales, P. (2006). Medición de actitudes en psicología y educación: construcción de escalas y problemas metodológicos. Madrid: Universidad Pontificia de Comillas.

Moreno, M. I. (1997). La formación básica del entrenador deportivo en la edad escolar. En M. A. Delgado (Ed.), Formación y actualización del profesorado de educación física y del entrenador deportivo (pp. 197-204). Wanceulen: Sevilla.

Moreno, M. P., \& Del Villar, F. (2004). El entrenador deportivo: manual práctico para su desarrollo y formación. Barcelona: Inde.

Nelson, L. J., Cushion, C. J., \& Potrac, P. (2006). Formal, non-formal and informal coach learning: A holistic conceptualisation. International Journal of Sports Science and Coaching, 1(3), 247-259.

Nuviala, A. (2003). Las escuelas deportivas en un entorno rural aragonés: el caso del Servicio Comarcal Ribera Baja. Zaragoza: Diputación General de Aragón.

Nuviala, A., León, J. A., Gálvez, J., \& Fernández, A. (2007). Qué actividades deportivas escolares queremos. Qué técnicos tenemos. Revista Internacional de Medicina y Ciencias de la Actividad Física y el Deporte, 7(25), 1-9. Recuperado de: http://cdeporte.rediris.es/revista/revista25/arttecdeport39.htm

Orts, F. J. (2013). El derecho educativo del menor en el deporte escolar. Tesis Doctoral. Universitat de Lleida, Lleida. Recuperado de: http:/hdl.handle.net/ $10803 / 112028$

Parker, T. (2000). Professional developments in exercise and sport sciencie: An Australian perspective. ICSSPE Bulletin, 30, 12-13.

Petitpas, A. J., Cornelius, A. E., Van Raalte, J. 1., \& Jones, T. (2005). A framework for planning youth sport programs that foster psychosocial development. The Sport Psychologist, 19(1), 63-80.

Petrus, A. J. (1996). Deporte escolar y nuevos derechos del niño. Pedagogía social: revista interuniversitaria, $14,7-30$

Pinillos, J. M. (2006). La educación Física y el deporte en Colombia: Una oposición de discursos en el periodo comprendido entre 1968 y 1991. Revista Educación Física y Deportes, 93. Recuperado de: http://www.efdeportes.com/efd93/ colombia.htm

Pozo, J. I., Scheuer, N., Pérez, M. P., Mateos, M., Martín, E., \& De La Cruz, M. (Eds.). (2006). Nuevas formas de pensar la enseñanza y el aprendizaje: Las concepciones de profesores y alumnos. Barcelona: Graó.

Rodríguez, J. M., Castillo, E., \& Jiménez, F. J. (2001). Necesidad de formación psicopedagógica de los entrenadores deportivos. Agora digital, 2. Recuperado de: http://dialnet.unirioja.es/servlet/extart?codigo=963218

Saura, J. (1996). El entrenador en el deporte escolar. Lleida: Fundació Pública Institut d'Estudis Llerdencs.

Velázquez, R. (2002). Educación deportiva y desarrollo moral: algunas ideas para la reflexión y para la práctica. Tándem: Didáctica de la educación física, 7, 7-20.

Velázquez, R., Devís, J., de la Torre, E., Carranza, M., \& Fraile, A. (2004). Debate sobre algunos tópicos del deporte escolar en España. En A. Fraile (Ed.), El deporte escolar en el siglo XXI: Análisis y debate desde una perspectiva europea (pp. 183-206). Barcelona: Graó.

Wenner, L., \& Jackson, S. (2009). Sport, beer, and gender in promotional culture: On the dynamics of a holy trinity. En L. Wenner \& S. Jackson (Eds.), Sport, beer, and gender: Promotional culture and contemporany social life (pp. 135). New York: Peter Lang. 